\title{
REVIEW
}

\section{Using green fluorescent protein to study intracellular signalling}

\author{
J M Tavaré, L M Fletcher and G I Welsh \\ Department of Biochemistry, School of Medical Sciences, University of Bristol, Bristol BS8 1TD, UK \\ (Requests for offprints should be addressed to J M Tavaré; Email: j.tavare@bristol.ac.uk)
}

\begin{abstract}
Subcellular compartmentalisation of signalling molecules is an important phenomenon not only in defining how a signalling pathway is activated but also in influencing the desired physiological output of that pathway (e.g. cell growth or differentiation, regulation of metabolism, cytoskeletal changes etc.). Biochemical analyses of protein and lipid compartmentalisation by, for example, subcellular fractionation presents many technical difficulties. However, this aspect of cell signalling research has seen a major revolution thanks to the cloning and availability of a variety of mutant green fluorescent protein derivatives with distinct molecular properties. Mutants with increased
\end{abstract}

brightness, altered excitation and emission maxima, altered stability and differential sensitivity to $\mathrm{pH}$, are now in widespread use for following the trafficking and function of proteins in living cells and for monitoring the intracellular environment. In this review we focus on some of the recent developments in the use of green fluorescent proteins for studying intracellular signalling pathways often with special reference to the actions of insulin. We also discuss the future utility of these proteins to analyse protein-protein interactions in signalling pathways using fluorescence resonance energy transfer.

Journal of Endocrinology (2001) 170, 297-306

\section{Introduction}

Green fluorescent proteins (GFPs) are found in a variety of bioluminescent organisms ranging from the jellyfish Aequorea victoria, to the IndoPacific sea anemone relative Discosoma (Tsien 1998, 1999, Matz et al. 1999). The advantages that these proteins confer on their parent organisms is not well understood, however they have been of undeniable benefit to thousands of cell biologists!

The major advantage of GFPs to the cell biologist is their ability to exhibit intrinsic fluorescence; for most other fluorescent proteins a cofactor is required. This property is endowed on GFP thanks to three amino acids that cyclise $\left(\operatorname{Ser}_{65}-\mathrm{Tyr}_{66}-\mathrm{Gly}_{67}\right)$ and then undergo an oxidation step during a complex maturation process. This fluorophore is deeply buried within the hydrophobic core of GFP that is, itself, made up of a compact barrel of eleven $\beta$-sheets with a central $\alpha$-helix containing the fluorophore. A detailed discussion of the physicochemical properties of GFPs is beyond the scope of this review and the reader is directed to a recent review which covers this topic in depth (Tsien 1998).

Wild-type Aequorea GFP has two excitation maxima at 395 and $470 \mathrm{~nm}$, and a single major emission peak at $509 \mathrm{~nm}$ (Table 1). Significant problems with this original variety of GFP were its weak signal intensity and some complex photochemical properties such as photoisomerisation. However, mutant forms of GFP with more stable physicochemical properties and, importantly, altered excitation/emission spectra have been developed that have really provided the impetus for a revolution in cell biology. Now there are varieties of Aequorea GFP that are blue, sapphire, cyan, yellow as well as a green version with simpler spectral properties (see Table 1). Most of these have now been mutated to encompass human codon usage rather than that for Aequorea. All these varieties are brighter and more photostable than the original wild-type GFP except, perhaps, for the blue fluorescent protein (BFP) which photobleaches very rapidly. However, under two-photon excitation, BFP is surprisingly photostable (J M Tavaré, unpublished data).

The introduction of epitope tags onto proteins has long been a valuable way of assessing the subcellular distribution of gene products (by using standard immunofluorescent techniques on fixed and permeabilised cell specimens using a specific antibody raised against the epitope, e.g. the haemagglutinin (HA) epitope). This is useful for assessing the spatial, but not temporal, aspects of protein expression. What is unique about GFP is its ability to allow the cell biologist to monitor the dynamics of GFP-tagged proteins in living cells, thus addressing both the spatial and temporal aspects. However, given that GFP is a $27 \mathrm{kDa}$ protein the investigator needs to be wary of deleterious effects of such a large protein tag on the 
Table 1 Spectral properties of GFPs. The properties of several variants of Aequorea victoria GFP that have been made commercially available or published in the literature are shown. The table includes values for excitation maximum $\left(\lambda_{\text {ex }}\right)$, emission maximum $\left(\lambda_{\text {em }}\right)$ and extinction coefficient $(\varepsilon)$. Values displayed in brackets indicate secondary excitation or emission peaks, or their respective extinction coefficients; N/A indicates that information is not available

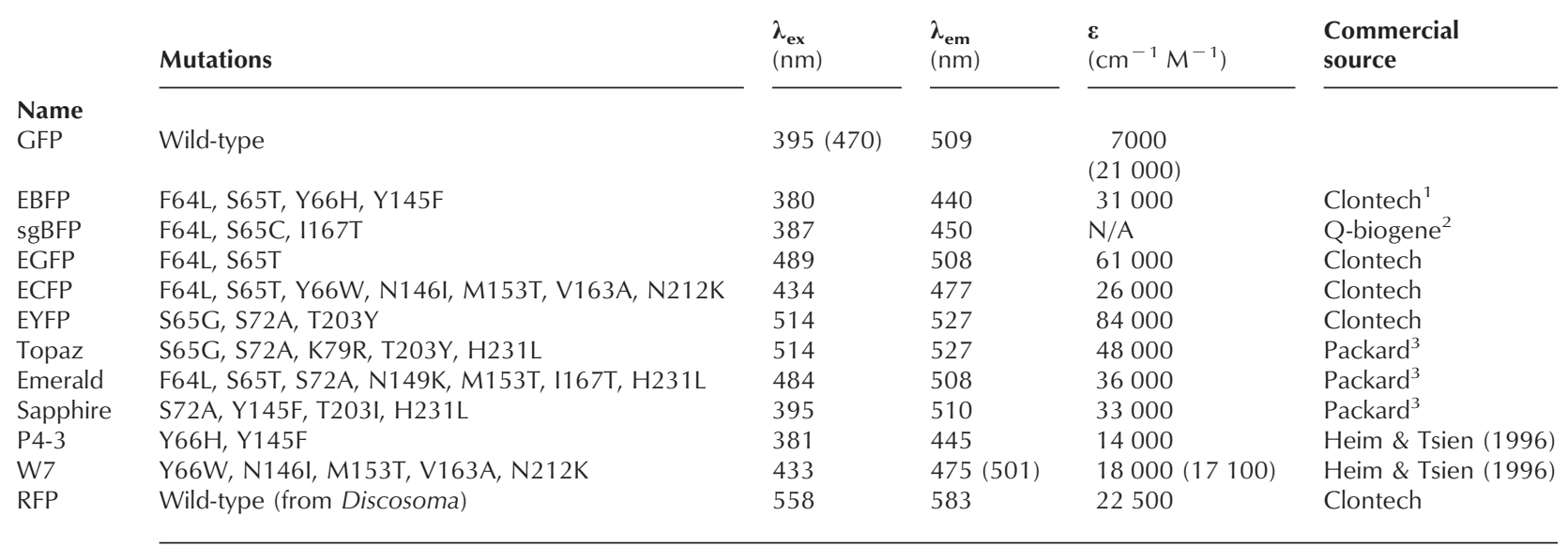

${ }^{1}$ Clontech Laboratories Ltd, Basingstoke, Herts, UK.

${ }^{2}$ Q-biogene, Harefield, Middx, UK.

${ }^{3}$ The GFPs from Packard are no longer commercially available.

function and localisation of the tagged protein. Despite this, the fact that the technique has been used successfully for well over a hundred proteins means that GFP is actually a remarkably versatile tag.

Using GFP allows the investigator to examine protein distribution in the living unadulterated cell, thus avoiding potential artefacts due to cell fixation and permeabilisation. On the other hand, GFP-tagged proteins are often expressed at levels far greater than the endogenous native protein and thus alterations in protein localisation and function due to over-expression should always be carefully considered and excluded using a series of judicious controls. Such controls would include ensuring that the GFP-tagged protein colocalises to the same compartment as the endogenous protein, and ensuring that the GFPtagged protein retains the function and characteristics expected of the native protein at both high and low levels of expression. With respect to the latter point, we often prefer to image the cells that express low levels of GFP-fusion proteins, in other words those cells where fluorescence is just detectable.

The detection limit of GFP-fusion proteins depends on many variables including the sensitivity of the detection system employed, the fluorescence intensity of the GFP in the cells used (which can vary considerably between cell types), whether the fusion protein is concentrated in subcellular compartments, and the degree of autofluorescence of the cells. Detection limits for GFPs have been reported to be approx. $10^{5}$ molecules per cell (Tsien 1998) but may come down to $10^{4}$ per cell if the protein is concentrated in subcellular compartments such as lipid rafts, intracellular vesicles or nucleoli.
The availability of different coloured GFPs allows two proteins tagged with distinct spectral variants to be localised in cells simultaneously. The recent cloning of the Discosoma red fluorescent protein (dsRFP) which is substantially red shifted compared with the Aequorea GFPs (Table 1), may herald a new avenue of exploration in cell biology - perhaps by allowing three GFP-tagged proteins to be individually localised in a single living cell. However, while dsRFP is undoubtedly bright it currently suffers from problems with homo-oligomerisation (Baird et al. 2000) thus greatly limiting its current value in cell biology.

In the first part of this review we will illustrate how the GFPs have been used to study the dynamics of signalling proteins. GFPs have been used to study a broad variety of different processes which have been reviewed in detail in many recent excellent reviews (e.g. see Tsien 1998, Bastiaens \& Pepperkok 2000, Chiesa et al. 2001). GFPs have been used to look at the movements of many proteins in real-time including transcription factors to and from the nucleus (e.g. NFkappaB (Tenjinbaru et al. 1999) and forkhead transcription factors (Takaishi et al. 1999)), movements of intracellular vesicles (such as pre-Golgi vesicles (Presley et al. 1997), secretory vesicles (Wacker et al. 1997) and peroxisomes (Monosov et al. 1996)), and microtubule dynamics (Perez et al. 1999).

A technique called fluorescence recovery after photobleaching (FRAP) has become increasingly used to study protein mobility in living cells. GFP-tagged proteins within a specific region of a cell (e.g. small regions of the Golgi complex) are bleached using a high power laser and the rate at which fluorescence recovers in this bleached region is related to the ability of non-bleached proteins to 


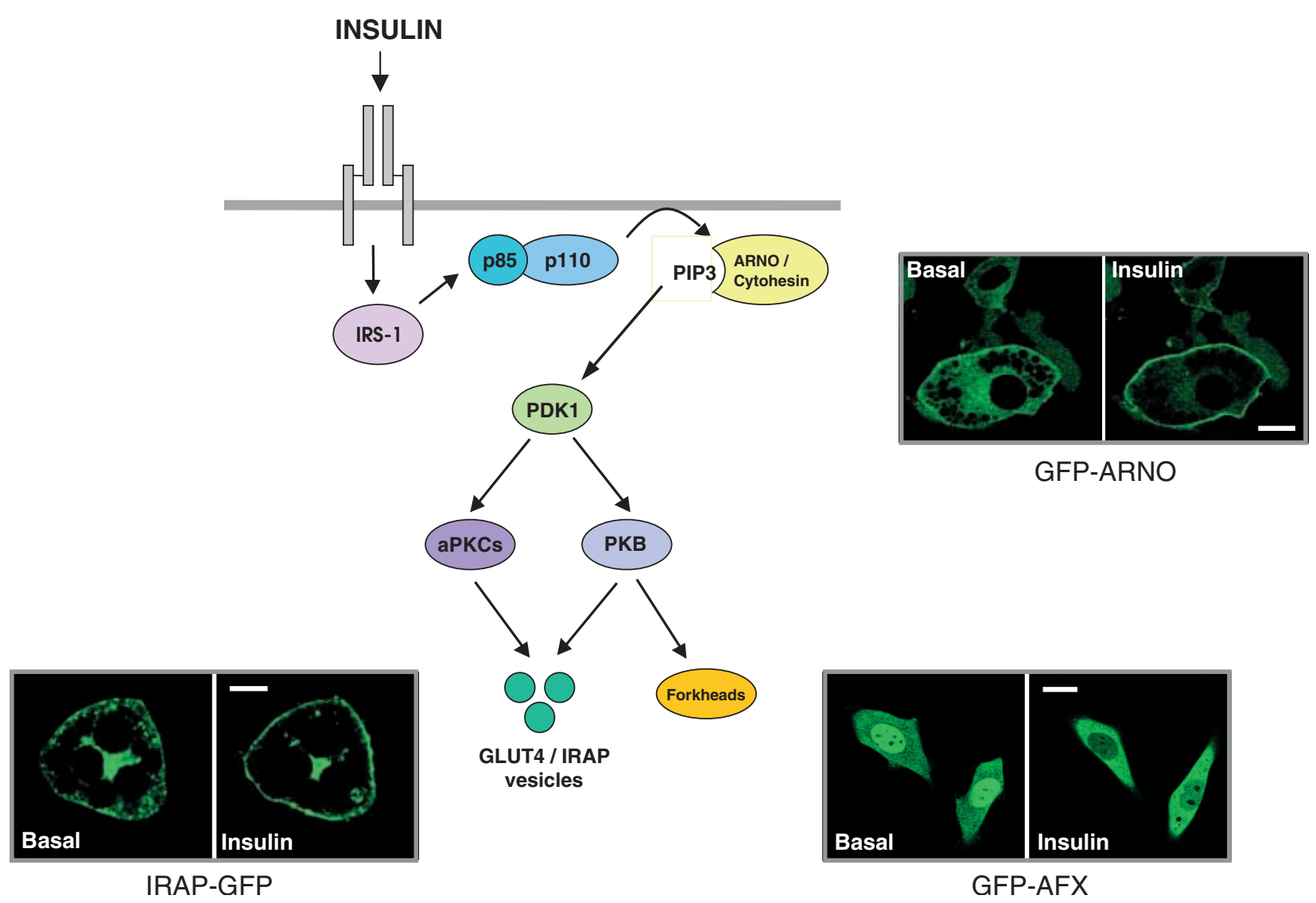

Figure 1 Insulin signalling pathways that have been studied using green fluorescent protein. See on-line version of this figure for animations of individual phenomena described in this review. The scale bar on each image represents $10 \mu \mathrm{m}$. Connect to http://www.bch.bris.ac.uk/staff/tavare/animation/signal.html

move back into that area. This has been particularly successfully used to study protein dynamics in the Golgi region and the nucleus (Storrie et al. 1998, Houtsmuller \& Vermeulen 2001).

As the field has now become so vast, in this review we will focus on how it has been used to study insulin signalling (specifically protein trafficking and lipid metabolism). However, an increasingly important use of the GFPs is going to be in allowing investigations into the temporal and spatial aspects of protein-protein interactions. Given the fact that $46 \%$ of the Helicobacter pylori proteome is estimated to be involved in heterologous protein-protein interactions (Rain et al. 2001) then this is going to be an increasingly important area of investigation over the next few decades. The final part of this review will also discuss the methods by which GFPs can be used to study these phenomena and how this may help drive future research.

\section{Using GFP to study protein trafficking}

The use of GFP has allowed the localisation and trafficking of proteins to be directly detected in a living cell in real time using time lapse fluorescence microscopy. This has overcome the need to fix and stain cells with specific antibodies to the protein of interest, a method which is open to possible fixation artefacts. To illustrate how the GFPs can contribute to our understanding of the dynamics and organisation of a signalling system, we shall use insulin receptor signalling as an example as summarised in Fig. 1. The figure is available in animated format at http:// www.bch.bris.ac.uk/staff/tavare/animation/signal.html.

Binding of insulin to its receptor results in activation of the lipid kinase phosphoinositide 3-kinase (PI3K) at the plasma membrane (Alessi \& Downes 1998). Activation of this enzyme generates a number of phosphorylated phosphatidylinositol (PI) intermediates such as PI(3,4,5)trisphosphate $\left(\mathrm{PIP}_{3}\right)$ which lead to the recruitment of a number of downstream signalling molecules to the plasma membrane through their ability to bind these PI intermediates (see also next section). One such signalling intermediate which translocates to the plasma membrane in response to the activation of $\mathrm{PI} 3 \mathrm{~K}$ is protein kinase $\mathrm{B}$ (PKB) which has been shown to be a key enzyme in insulin signalling (Fig. 1; reviewed in Alessi \& Downes 
1998, Coffer et al. 1998, Vanhaesebroeck \& Alessi 2000).

The translocation of PKB to the plasma membrane results in its phosphorylation and activation by an upstream serine/threonine protein kinase called PDK-1. GFPtagged PKB constructs have been used to show that the enzyme translocates to the plasma membrane in response to insulin, platelet-derived growth factor (PDGF), epidermal growth factor (EGF) and serum. The translocated PKB becomes highly concentrated in plasma membrane ruffles, consistent with the localised concentration of $\mathrm{PIP}_{3}$ in these regions; this phenomenon is difficult to visualise in fixed cells (see http://www.bch.bris.ac.uk/staff/tavare/ animation/signal.html and link therein for animation of this process). The translocation of PKB to the plasma membrane is dependent on the activation of PI3K and on the PKB having an intact pleckstrin homology $(\mathrm{PH})$ domain, since mutation of even a single amino acid in the $\mathrm{PH}$ domain necessary for $\mathrm{PIP}_{3}$ binding blocked membrane translocation (Andjelkovic et al. 1997, Watton \& Downward 1999, Vanhaesebroeck \& Alessi 2000). Recent evidence using GFP-tagged PKB has demonstrated that an intact actin cytoskeleton is necessary for PI3K activity and hence for the activation of PKB by hormones and growth factors (Peyrollier et al. 2000).

The localisation of PDK-1, the upstream activator of PKB, has also been studied by GFP tagging. However, so far, this has not produced a clear picture. It had been reported that PDK-1 translocates from the cytoplasm to the plasma membrane in response to PDGF and that this was important in allowing PDK-1 to activate PKB (Anderson et al. 1998). However, a more recent study using the same cells has suggested that PDK-1 is localised constitutively at the plasma membrane and that the translocation seen previously reflects formation of new plasma membrane structures on addition of the hormone or growth factor (Currie et al. 1999). In the latter study, quantitative immunogold electron microscopy was used to compliment experiments using GFP-tagged PDK-1. These differences demonstrate that while GFP tagging is a very powerful method, the results need to be confirmed by other biochemical techniques. It may, however, also highlight the strong possibility that signalling systems operate in a cell type specific manner.

GFP tagging has also been used to study cellular events downstream of PKB. For example, the use of GFP-tagged proteins has been ideally suited to studying the mechanisms by which insulin stimulates glucose transport. An increase in the rate of glucose transport in muscle and adipose tissue is one of the major downstream responses following insulin stimulation. This results primarily from a rapid insulin-dependent translocation of GLUT4 (the insulin-responsive glucose transporter isoform) from intracellular vesicular storage sites to the plasma membrane. Experiments using GFP-tagged GLUT4 have shown that this protein is found in at least two inter-related vesicle populations: endosomes and a specialised 'GLUT4 vesicle' compartment which also contains an insulinresponsive amino peptidase (IRAP). Furthermore, translocation of both populations of GLUT4 vesicle is dependent on the activation of PI3K whereas only that of the 'GLUT4 vesicle' pool to the plasma membrane can be induced by constitutively active forms of PKB (Foran et al. 1999). The insulin-dependent translocation can be visualised by time-lapse confocal microscopy of either GFPGLUT4 (Fletcher et al. 2000) or IRAP-GFP (see Fig. 1).

Recently, using GFP-GLUT4 and time lapse confocal microscopy we have shown that there is a role for the microtubule cytoskeleton in 'GLUT4 vesicle' trafficking and in the regulation of insulin-stimulated glucose uptake (Fletcher et al. 2000). For example, close examination of the on-line animation of GFP-GLUT4 associated with Fig. 1 reveals rapid linear movements of GFP-GLUT4 vesicles indicative of motor-driven movements along microtubules. Indeed these movements are completely blocked by microtubule depolymerisation agents (see Fletcher et al. 2000 for a more detailed animation of this process). The majority of vesicles, however, exhibit rapid vibratory motions around a point rather than the linear excursions just alluded to. Clearly these vesicles are tethered to an intracellular structure that prevents free movement. This structure is not the cytoskeleton as the vibrations are not disrupted by actin or microtubule depolymerising agents.

The studies on GFP-tagged GLUT4 vesicles probably demonstrate the greatest utility for GFP - its use for dynamic imaging. The dynamic phenomena described above would be impossible to reveal using more traditional biochemical or static cell biological techniques such as immunofluorescence microscopy.

$\mathrm{PKB}$ has also been shown to regulate the activity of members of the human forkhead transcription family, including AFX. The forkhead family of transcription factors all contain three sites which are substrates for phosphorylation by PKB. There is also a great deal of evidence suggesting that they lie downstream of, and their activity is negatively regulated by, the PI3K/PKB pathway (Kops \& Burgering 1999, Takaishi et al. 1999). As shown in the on-line version of Fig. 1, we can visualise the translocation of a GFP-tagged AFX construct from the nucleus to the cytoplasm upon activation of PKB. This translocation is blocked by mutagenesis of the PKB phosphorylation sites on AFX, by inhibitors of PI3K activation such as wortmannin, or by dominant-negative forms of either PI3K or PKB (Takaishi et al. 1999). Thus PKB regulates the transcriptional activity of AFX by promoting its export from the nucleus. Once exported from the nucleus, AFX binds to members of the 14-3-3 family of proteins thus anchoring the transcription factor in the cytoplasm. 


\section{Using GFP to study lipid metabolism}

Agonist-stimulated generation of phosphoinositides is recognised to be a major route by which many growth factors convey signals to cellular effectors. Activation of class Ia PI3Ks by insulin and other growth factors stimulates rapid phosphorylation of phosphatidylinositol-4,5-bisphosphate $\left(\mathrm{PI}(4,5) \mathrm{P}_{2}\right)$ to yield a marked increase in $\mathrm{PIP}_{3}$ and, via the subsequent action of a $5^{\prime}$-phosphatase, phosphatidylinositol-3,4-bisphosphate $\left(\mathrm{PI}(3,4) \mathrm{P}_{2}\right)$. Both $\mathrm{PIP}_{3}$ and $\mathrm{PI}(3,4) \mathrm{P}_{2}$ play a crucial role in a variety of cellular processes including control of glucose uptake and glycogen synthesis, cytoskeletal rearrangements, vesicle trafficking, and cell survival. Alternatively, cellular agonists which activate phospholipase C (PLC) mediate cleavage of $\mathrm{PI}(4,5) \mathrm{P}_{2}$, generating the second messengers diacylglycerol (DAG) and inositol-3,4,5-trisphosphate $\left(\mathrm{IP}_{3}\right)$. Subsequent $\mathrm{IP}_{3}$-mediated release of $\mathrm{Ca}^{2+}$ from internal endoplasmic reticulum stores activates many events including neurotransmitter release.

One of the major mechanisms by which lipid effectors transmit signals is via the recruitment, or translocation, of a range of signalling proteins containing $\mathrm{PH}$ domains to various membrane compartments. Indeed, many of the key molecular players downstream of the insulin receptor are $\mathrm{PH}$-domain containing proteins, including insulin receptor substrate (IRS) proteins, PKB and PDK-1 (see Fig. 1). Although $\mathrm{PH}$ domains from different proteins are structurally related, their affinity for different PIs varies significantly. For example, the PH domain of the small guanine-nucleotide exchange factor ARNO is highly specific for $\mathrm{PIP}_{3}$, whereas the $\mathrm{PH}$ domain of PLC $\delta 1$ binds in preference to $\mathrm{PI}(4,5) \mathrm{P}_{2}$, as judged by in vitro binding assays. Based on such properties, investigators have successfully utilised fusions between GFP and PH domains, in combination with rapid frame-rate confocal microscopy, as sensitive in vivo probes for the generation of specific phospholipids. Such studies have allowed monitoring of changes in phospholipid levels in single living cells over a short (seconds) time-range, revealing both dynamic and spatial aspects of lipid signalling that are close to impossible to achieve using traditional techniques of subcellular fractionation and HPLC analysis of phospholipids.

GFP-ARNO, for example, is localised exclusively to the cytoplasm in resting 3T3-L1 adipocytes but translocates rapidly (within approx. $40 \mathrm{~s}$ ) to the cell periphery upon insulin stimulation, reflecting the production of $\mathrm{PIP}_{3}$ at the plasma membrane (as illustrated in Fig. 1 and its online supplement; Venkateswarlu et al. 1998b, Oatey et al. 1999). A similar phenomenon is observed using the $\mathrm{PH}$ domains of the related proteins general receptor for phosphoinositides-1 (GRP-1) or cytohesin (Venkateswarlu et al. 1998a, 1999, Oatey et al. 1999). The $\mathrm{PH}$ domains of $\mathrm{PKB}$ and Brutons tyrosine kinase (Btk), which bind both $\mathrm{PI}(3,4) \mathrm{P}_{2}$ and $\mathrm{PIP}_{3}$, have also been used to follow the plasma membrane generation of these 3-phosphorylated phosphoinositides in a similar manner (Gray et al. 1999, Varnai et al. 1999).

In another study, Meyer and colleagues utilised the $\mathrm{PH}$ domain of PLC $\delta 1$ fused with GFP to demonstrate a transient PLC $\gamma$-mediated reduction of $\mathrm{PI}(4,5) \mathrm{P}_{2}$ in the plasma membrane of rat basal leukemia cells stimulated with platelet activating factor (Stauffer et al. 1998). However, an important point to raise here is that $\mathrm{PH}$ domains do not display exclusive selectivity for one particular PI. In addition to binding $\mathrm{PI}(4,5) \mathrm{P}_{2}$, the $\mathrm{PH}$ domain of PLC $\delta 1$ also has a high affinity for $\mathrm{IP}_{3}$. Indeed, Hirose et al. (1999) provided evidence that dissociation of GFP-PLC $\delta 1 \mathrm{PH}$ from the plasma membrane in ATP-stimulated MDCK cells was instead mediated by an increase in cytosolic $\mathrm{IP}_{3}$ levels that displace $\mathrm{PI}(4,5) \mathrm{P}_{2}$ from the PLC $\delta 1 \mathrm{PH}$ domain. In this study, time-lapse imaging during ATP stimulation revealed oscillations of $\mathrm{IP}_{3}$ generation that coincided with $\mathrm{Ca}^{2+}$ waves monitored using fura-2 (Hirose et al. 1999). Clearly, therefore, in order to use GFP-PLC $\delta 1 \mathrm{PH}$ to monitor $\mathrm{PI}(4,5) \mathrm{P}_{2}$, the conditions must favour PI-4,5- $\mathrm{P}_{2}$ binding rather than $\mathrm{IP}_{3}$. This requires controls using cell permeant $\mathrm{IP}_{3}$ analogues.

Therefore, it is a necessary consideration for investigators that there may be more than one PI contributing to the localisation of a GFP-PH domain probe, and that the precise levels of different PIs generated are likely to vary significantly between different stimuli and cell lines. An additional problem when monitoring changes in subcellular localisation of GFP-PH fusions using confocal microscopy techniques is that cellular stimulation often results in considerable changes in cell morphology and membrane structure (e.g. formation of membrane ruffles). This presents difficulties when quantifying translocation in a region of interest over time. Additionally, in very flat cells such as fibroblasts, it is very difficult to visualise the plasma membrane using fluorescence- or confocal-based microscopes. For these reasons, a recent study has turned to the use of a fluorescence resonance energy transfer (FRET)-based assay for phospholipid generation (the use of FRET is described in more detail in the following section).

van der Wal and co-workers (2001) have described the use of FRET between PLC $\delta 1 \mathrm{PH}$ domains tagged with CFP or yellow fluorescent protein (YFP) to monitor PLC $\gamma$ activation using wide-field optical detection. This method calculates fluorescence emission from an entire cell (thereby negating the influence of cell shape changes). Under basal conditions, CFP-PLC $\delta 1 \mathrm{PH}$ and YFPPLC $\delta 1 \mathrm{PH}$ localise largely to the plasma membrane of cells, and display FRET between the two fluorophores. Upon cellular stimulation, membrane dissociation of the fusion constructs results in a measurable decrease in FRET. It is likely that such techniques will be applicable to a wide variety of $\mathrm{PH}$ domain-based probes, allowing rapid and sensitive monitoring of a range of phospholipid signalling molecules. 
An emerging theme from the studies described above, and others, is that the location and magnitude of phospholipid generation may be a major factor in determining the precise response of a cell to a particular stimulus. For example, although both insulin and PDGF are capable of activating whole cell PI3K to a similar extent in 3T3-L1 adipocytes, using ARNOPH-GFP as a probe we observed that insulin stimulates the generation of plasma membrane $\mathrm{PIP}_{3}$ to a greater degree and in considerably more cells (Oatey et al. 1999). This may provide a possible explanation for the greater ability of insulin to activate PKB and cause GLUT4 translocation.

Spatial regulation of lipid signalling within the plasma membrane is also likely to be important. In this vein, motile fibroblasts stimulated with a PDGF concentration gradient are seen to generate local $3^{\prime}$-phosphoinositides (monitored using a GFP-PKBPH fusion) in regions of membrane adhesion and spreading (Haugh et al. 2000). In macrophages, chimeras of GFP with PLC $\delta 1 \mathrm{PH}$ or with the $\mathrm{C} 1$ domain of protein kinase $\mathrm{C} \delta(\mathrm{PKC} \delta$ ) (which has a high affinity for DAG) have been employed to demonstrate localised accumulation of $\mathrm{PI}(4,5) \mathrm{P}_{2}$ and $\mathrm{DAG}$ in plasma membrane regions undergoing phagocytosis (Botelho et al. 2000).

In a quite beautiful dynamic study, a protein called DAPP1, which contains a C-terminal PH domain, translocates from the cytosol to the plasma membrane in porcine endothelial cells stimulated with PDGF (Anderson et al. 1998). After this, DAPP1 was endocytosed into early endosomes which contained internalised PDGF receptors. This phenomenon would be almost impossible to visualise without the advent of GFP technology.

Spatial modes of regulation, such as those described above, may turn out to be of importance from an endocrinologist's viewpoint; for example, recent studies have highlighted the potential importance of localised 'lipid rafts' in insulin signalling to glucose uptake. These are areas of the plasma membrane enriched in caveolin, cholesterol and $\mathrm{PIP}_{2}$ which recruit a complex between the proteins $\mathrm{Cbl}$ and $\mathrm{c}-\mathrm{Cbl}$-associating protein (CAP); disruption of complex formation significantly attenuates insulinstimulated GLUT4 translocation (Baumann et al. 2000).

\section{Using GFP to study protein-protein interactions in living cells}

The transmission of a signal through a signalling pathway involves a large variety of different types of protein-protein interaction including those that are weak and transient (i.e. the phosphorylation of a substrate protein by a protein kinase) or strong and stable (i.e. the interaction between a tyrosine phosphorylation site on a receptor and its cognate SH2 domain). In many cases the intracellular location of these sorts of interaction are completely unknown, with their analysis relying on techniques such as coprecipitation (which will only reveal strong stable

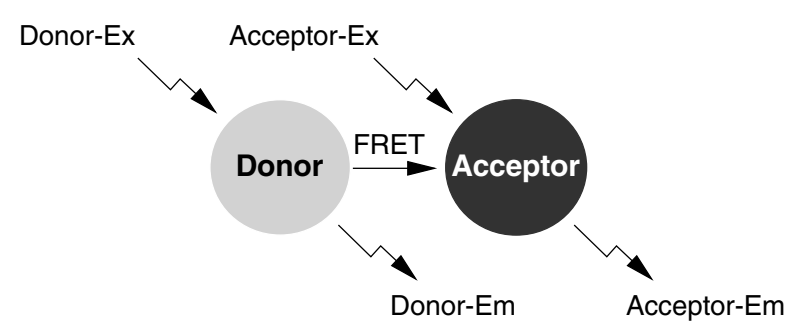

Figure 2 Using FRET and green fluorescent protein to measure protein-protein interactions. FRET between the donor and acceptor fluorophores can be measured in a number of ways using fluorescence microscopy. (i) Ratio of Donor to Acceptor fluorescence emission intensities (Donor-Em/Acceptor-Em). (ii) FRETN, a measure of FRET corrected for changes in local Donor and Acceptor concentrations (i.e. corrects for increases in Donor-Em and Acceptor-Em signals due to non-FRET phenomena). (iii) FLIM, a measure of the picosecond-nanosecond excited state lifetime of the Donor (i.e. Donor-Em lifetime) which is decreased when an Acceptor is sufficiently close (i.e. $<50 \AA$ ) to induce FRET. (iv) Donor quenching imaging, measures the increase in Donor-Em intensity that occurs when the Acceptor is photobleached by flooding the sample with light at the Acceptor-Ex wavelength. (v) Donor photobleaching rate, measures the seconds to minute bleaching rate of the Donor which is caused by flooding the sample with light corresponding to the Donor-Ex wavelength. See text for further details.

interactions), or apparent co-localisation as detected by double immunofluorescence microscopy (i.e. using antibodies to immunostain each protein within a single sample). The resolution of the latter technique is limited by the optical resolution of the microscope as, in general, light microscopes cannot resolve objects that are less than $\sim 3000 \AA$ apart (even after extensive mathematical improvements of the raw image obtained). Given the diameter of a typical protein (say $<100 \AA)$ direct determination of protein-protein interactions is not possible using this technique.

To examine protein-protein interactions using fluorescence microscopy the investigator is required to turn to the use of fluorescence resonance energy transfer (FRET), a property that is exhibited by some of the GFP variants. Given the appropriate spectral properties, two fluors placed less than $\sim 50 \AA$ apart will exhibit FRET (see Fig. 2); i.e. the emitted light from one fluor will directly excite the second fluor (as long as the emission spectrum of the first fluor overlaps with the excitation spectrum of the second fluor). Several investigators have taken advantage of this complex biophysical property of the different coloured GFPs to investigate a variety of different interactions.

\section{Physicochemical properties of GFPs that lend themselves to studying protein-protein interactions}

Two pairs of GFPs have commonly been used to study protein-protein interactions in living cells using FRET (see Pollok \& Heim 1999 for a discussion of the selection criteria). These are: (i) blue (BFP) and green (GFP) 
fluorescent proteins, or (ii) cyan (CFP) and yellow (YFP) fluorescent proteins. The former pair is probably more suitable given the more optimal spectral overlap between BFP and GFP. However, BFP is only weakly fluorescent and rapidly photobleaches, thus greatly limiting its utility for most applications. Because the CFP/YFP pair is brighter, more photostable, and gives greater signal to noise, it is the more commonly used pair despite the fact that they have significantly less optimal spectral overlap (thus the observed changes in FRET intensity can be lower than with the BFP/GFP pairing). Finally, RFP and GFP have been used to study protein-protein interactions (Cornea et al. 2001) although care should be taken when using this pair given the oligomerisation problems exhibited by RFP (Baird et al. 2000).

\section{Methodologies commonly used to follow FRET in cells}

At this stage, it is worth mentioning the methodologies used to measure FRET because this represents the most crucial and difficult part of this powerful technology. Indeed, the methods range from relatively simple, off the shelf systems (which make interpretation more difficult) to the considerably more sophisticated but versatile home built systems for the officionado.

The simplest method involves measuring a ratio of donor to acceptor fluorescence emission intensities (see Fig. 2). This can be achieved with most conventional dual channel laser-confocal microscopes, or epifluorescence microscopes using a CCD camera and appropriate excitation/emission filter sets. Given appropriate controls (of which there are many), this technique will often prove satisfactory. However, the purpose of the controls is generally to correct for the data for (i) interfering background fluorescence, (ii) complications due to local changes in the concentration of the donor or acceptor, and (iii) the facts that: (a) the light used to excite the donor usually also excites the acceptor, at least to a degree, and (b) that the emission spectrum of the donor often overlaps significantly with the emission spectrum of the acceptor.

There are two highly complex methods of avoiding these problems and these are called FRETN and FLIM (reviewed in Gordon et al. 1998, Bastiaens \& Squire 1999). FRETN is a mathematical manipulation of the type of ratiometric data collected above but which additionally allows the investigator to correct for background fluorescence and for the degrees to which the donor and acceptor excitation and emission spectra overlap (Gordon et al. 1998). This method is suitable for studies on living cells and can be applied using most conventional fluorescence microscopes.

FLIM essentially measures a change in the 'fluorescence lifetime' of the donor fluor. When a donor fluor is excited by a photon, its excited state decays within a few picoseconds. This fluorescence lifetime is reduced when an acceptor fluor is placed within sufficiently close proximity to the donor to exhibit FRET (often $<50 \AA$ ) and can be measured using fluorescence lifetime imaging (FLIM; Bastiaens \& Squire 1999, Bastiaens \& Pepperkok 2000). One major advantage of FLIM is that likely changes in the local concentration of the fluor are automatically taken account of. However, the major setback is a complex technical one - the method requires a complex pulsing or modulating light source and there are no commercially available systems currently on the market.

Two other rather simpler microscope-based methods are available. One, termed donor quenching imaging, relies on the fact that by bleaching the acceptor (achieved by flooding the sample with light corresponding to the acceptor's excitation wavelength), an increase in the fluorescence emitted by the donor occurs (because FRET can no longer take place due to an obliteration of the acceptor fluorophore). However, the result is that the method cannot be applied to living cells since it is also highly toxic to those cells.

In a final related method, FRET with an acceptor will cause a reduction in the rate at which the fluorescence of the donor photobleaches (a phenomenon which occurs over seconds to minutes). However, again, this technique is not suitable for live cell imaging due to a permanent destruction of the fluorophore.

\section{Protein-protein interactions can be studied using FRET between GFP derivatives}

The technique of FRET has yet to be successfully applied to any protein-protein interaction system in the insulin signalling pathway. So, in the following section we will provide a general discussion of the signalling systems that have been successfully studied.

FRET between BFP and GFP was first successfully used to generate a protease biosensor. BFP was directly fused to GFP using a short peptide linker containing either a tryptic or factor X cleavage sequence thus causing BFP and GFP to interact and exhibit FRET (Heim \& Tsien 1996, Mitra et al. 1996). When the purified fusion protein was incubated with the protease, a reduction in FRET was observed. This method was subsequently adapted by introducing a caspase-3 cleavage sequence into the linker, although a CFP-YFP pair was used which is a considerably brighter reporter fusion (as discussed above). Introduction of this fusion protein into cells allows the activation of caspase- 3 to be followed in real-time in single cells undergoing apoptosis (Xu et al. 1998, Mahajan et al. 1999, Tyas et al. 2000).

FRET between GFPs has also been used to monitor the cellular concentration of two important small molecule second messengers, cAMP and $\mathrm{Ca}^{2+}$. To generate a reporter for cAMP levels in single living cells, BFP was fused to the regulatory subunit of cAMP-dependent protein kinase, and GFP to the catalytic subunit (Zaccolo et al. 2000). A decrease in FRET was observed after incubating cells expressing the reporter with norepinephrine, 
isoproterenol or forskolin (due to the binding of the raised cAMP to the regulatory subunit and thus dissociation of the regulatory and catalytic subunits of the kinase).

$\mathrm{Ca}^{2+}$ levels can be measured using reporters called Cameleons. Here, CFP is fused to YFP either (i) directly via a spacer peptide containing both calmodulin and the calmodulin-binding peptide M13, or (ii) by separately fusing CFP to calmodulin, and YFP to the M13 peptide, as individual fusion proteins (Miyawaki et al. 1997). This genetically encoded $\mathrm{Ca}^{2+}$ biosensor has been used to study changes in $\mathrm{Ca}^{2+}$ ion concentrations in a variety of cell types. Increased binding of $\mathrm{Ca}^{2+}$ induces an increase in FRET as a result of an interaction of CFP-calmodulin with YFP-M13. Being genetically encoded sensors, the Cameleons can be targeted to specific intracellular compartments to report changes in local $\mathrm{Ca}^{2+}$ concentrations. This has been achieved, for example, by targeting the Cameleons to the endoplasmic reticulum, nucleus or to secretory vesicle (Miyawaki et al. 1997, Emmanouilidou et al. 1999, Pollok \& Heim 1999).

A small number of protein-protein interactions have been studied using FRET between the different spectral variants of GFP. This includes an interaction between the transcription factors Pit-1 and c-Ets-1 (Day 1998), which control prolactin gene expression. In another study, an interaction between $\mathrm{Bcl}-2$ and $\mathrm{Bax}$, two mitochondrial proteins involved in apoptosis, was also observed using FRET microscopy (Mahajan et al. 1998). As would be predicted, the FRET signal was only observed when Bcl-2-BFP was co-expressed with Bax-GFP and not when Bcl-2-BFP was co-expressed with cytochrome c-GFP, two proteins which are known not to interact. In neither of these studies was any regulation of the interaction attempted. For example, the interaction between Bcl-2 and Bax is well known to be reduced by a variety of apoptotic insults, although this has yet to be demonstrated using FRET microscopy.

An agonist-induced change in protein-protein interaction has been studied in three cases to date. The first case, and one of particular interest to endocrinologists, is the recent demonstration that gonadotrophin-releasing hormone receptor $(\mathrm{GnRH})$ microaggregation can be imaged using FRET microscopy (Cornea et al. 2001). Here, the GnRH was fused at the C-terminus with either GFP or RFP. The GnRH-GFP and GnRH-RFP fusion proteins were co-expressed in cells and an interaction monitored by measuring FRET between the GFP and RFP moieties. By measuring donor fluorescence after acceptor photobleaching, a small increase in GnRH/GnRH interaction could be visualised and this was interpreted as a ligand-induced receptor microaggregation. That this could be observed was remarkable given that only $50 \%$ of the interactions (i.e. the GnRH-GFP/GnRH-RFP 'hetero'dimers) could have exhibited FRET (the GnRH-GFP/ GnRH-GFP and GnRH-RFP/GnRH-RFP homodimers being unable to FRET).
In the second case, an interaction between soluble NSF attachment protein-23 (SNAP-23) and synaptobrevin has been reported by fusion with CFP and YFP respectively (Cornea et al. 2001). Neurosecretion in PC12 cells induced by $\mathrm{KCl}$ was reported to increase the interaction between these two soluble NSF attachment protein receptor (SNARE) proteins, as determined by an increase in FRET, although unexpectedly a significant interaction also occurred in the non-stimulated state.

The third case involves the EGF receptor (labelled with CFP on its cytosolic C-terminus) and its downstream effector Grb2 (labelled with YFP; Sorkin et al. 2000). Grb2-YFP interacts with the CFP-EGF receptor fusion in an EGF-dependent manner, and only when the correct tyrosine on the EGF receptor has become autophosphorylated. This interaction could be followed by FRET microscopy of PAE cells and, remarkably, the interaction was found to occur not just in the plasma membrane and membrane ruffles, but also within endosomes. The latter result is intriguing as it has been proposed over many years that ligand-bound receptors can be internalised in an 'active' state to intracellular sites such as endosomes, from where they can continue to signal. This represents one method by which a cell surface signal can be delivered to specific intracellular compartments.

\section{Using GFPs to monitor other physicochemical properties of proteins in cells}

FRET has been used to examine the interaction of GFP fusion proteins with other fluorescently labelled molecules, for example antibodies and other binding proteins. In this section we briefly describe methods that have used this technology to study signalling systems.

\section{Measurement of protein phosphorylation using GFP}

The autophosphorylation of the EGF receptor tyrosine kinase has been measured using FRET (Bastiaens \& Squire 1999). To do this the EGF receptor was tagged with GFP and expressed in cells which were subsequently stimulated with EGF to induce autophosphorylation in vivo and then fixed. The fixed cells were incubated with a Cy3-labelled anti-phosphotyrosine antibody. While this antibody would have bound all the tyrosine-phosphorylated proteins in the EGF-stimulated cells (i.e. Shc, Gab1, Erk etc.) only when it bound to the GFP-labelled EGF receptor was a FRET signal observed; all other interactions being invisible to the imaging system. A very similar trick has been developed to measure PKC phosphorylation and activation using a GFP-PKC fusion protein and Cy3-5-labelled phosphorylation site-specific antibodies that recognise the phosphorylation site on PKC that is responsible for its activation $(\mathrm{Ng}$ et al. 1999). This technique clearly has wide utility to measure the phosphorylation of almost any protein in a cell 
and is adaptable to measuring protein phosphorylation in living cells in real-time.

\section{Rac activation}

$\mathrm{Rac}$ is a small GTP binding protein of the Ras family and plays a diverse role in signalling including regulation of cell morphology and the actin cytoskeleton. Its activity state is governed by the extent of GTP binding. Once GTP has bound, Rac can then interact with downstream effector molecules such as the protein kinase, PAK1. The RacGTP-binding domain of PAK1 (PDB) was labelled with a fluorescent Alexa 546 dye and this was injected into cells expressing a functional Rac-GFP fusion protein (Rucktaschel et al. 2000). Rac activation was reported as FRET between Rac-GFP and PBD-Alexa 546. This was observed to occur in membrane ruffles and in the leading edge of motile cells that were spreading into an artificially induced wound site. Again, this is a technique that should be adaptable to other members of the small GTP-binding protein family, for which there is a known effector protein that binds in a nucleotide-specific manner.

\section{The future}

The studies described in this review represent only the beginning of the revolution in cell biology! There is no doubt that GFP has completely changed our perception of what is possible in cell signalling research. One future issue will be the size of GFP. Despite the fact that it is $27 \mathrm{kDa}$ and appears rarely to affect the subcellular localisation of the protein to which it is tagged, many controls are still required to ensure that the parent protein behaves as one would expect the native counterpart. To bypass this problem, we are already beginning to see the introduction of technologies to tag proteins with much smaller cellpermeant arsenical-based fluorescent compounds such as those used in the FLASH system developed by Tsien and colleagues (Griffin et al. 1998).

Another problem centres around the current requirement to over-express the tagged proteins and this can itself lead to a whole variety of potential artefacts. However, the technology to tag endogenous proteins by homologous recombination is now available and combined with the FLASH labelling system may help overcome this important limitation.

There can be little doubt that the past five years has seen a major revolution in cell biology and one can only wait with bated breath to see where the technology will take us over the next ten years.

\section{Acknowledgements}

Work in the authors' laboratory was supported by grants from The Medical Research Council, The Wellcome Trust and Diabetes UK. J M T is a Diabetes UK Senior Research Fellow.

\section{References}

Alessi DR \& Downes CP 1998 The role of PI 3-kinase in insulin action. Biochimica et Biophysica Acta 1436 151-164.

Anderson KE, Coadwell J, Stephens LR \& Hawkins PT 1998 Translocation of PDK-1 to the plasma membrane is important in allowing PDK-1 to activate protein kinase B. Current Biology 8 684-691.

Andjelkovic M, Alessi DR, Meier R, Fernandez A, Lamb NJ, Frech M, Cron P, Cohen P, Lucocq JM \& Hemmings BA 1997 Role of translocation in the activation and function of protein kinase B. Journal of Biological Chemistry 272 31515-31524.

Baird GS, Zacharias DA \& Tsien RY 2000 Biochemistry, mutagenesis, and oligomerization of DsRed, a red fluorescent protein from coral. PNAS 97 11984-11989.

Bastiaens PI \& Squire A 1999 Fluorescence lifetime imaging microscopy: spatial resolution of biochemical processes in the cell. Trends in Cell Biology 9 48-52.

Bastiaens PI \& Pepperkok R 2000 Observing proteins in their natural habitat: the living cell. Trends in Biochemical Sciences 25 631-637.

Baumann CA, Ribon V, Kanzaki M, Thurmond DC, Mora S, Shigematsu S, Bickel PE, Pessin JE \& Saltiel AR 2000 CAP defines a second signalling pathway required for insulin-stimulated glucose transport. Nature $\mathbf{4 0 7} 202-207$.

Botelho RJ, Teruel M, Dierckman R, Anderson R, Wells A, York JD, Meyer T \& Grinstein S 2000 Localized biphasic changes in phosphatidylinositol-4,5-bisphosphate at sites of phagocytosis. Journal of Cell Biology 151 1353-1368.

Chiesa A, Rapizzi E, Tosello V, Pinton P, De Virgilio ME, Fogarty K \& Rizzuto R 2001 Recombinant aequorin and green fluorescent protein as valuable tools in the study of cell signalling. Biochemical Journal 355 1-12.

Coffer PJ, Jin J \& Woodgett JR 1998 Protein kinase B (c-Akt): a multifunctional mediator of phosphatidylinositol 3-kinase activation. Biochemical Journal 335 1-13.

Cornea A, Janovick JA, Maya-Núñez G \& Conn PM 2001 Gonadotropin-releasing hormone receptor microaggregation. Journal of Biological Chemistry 276 2153-2158.

Currie RA, Walker KS, Gray A, Deak M, Casamayor A, Downes CP, Cohen P, Alessi DR \& Lucocq J 1999 Role of phosphatidylinositol 3,4,5-trisphosphate in regulating the activity and localization of 3-phosphoinositide-dependent protein kinase-1. Biochemical Journal 337 575-583.

Day RN 1998 Visualization of Pit-1 transcription factor interactions in the living cell nucleus by fluorescence resonance energy transfer microscopy. Molecular Endocrinology 12 1410-1419.

Emmanouilidou E, Teschemacher AG, Pouli AE, Nicholls LI, Seward EP \& Rutter GA 1999 Imaging $\mathrm{Ca}^{2+}$ concentration changes at the secretory vesicle surface with a recombinant targeted cameleon. Current Biology 9 915-918.

Fletcher LM, Welsh GI, Oatey PB \& Tavaré JM 2000 Role for the microtubule cytoskeleton in GLUT4 vesicle trafficking and in the regulation of insulin-stimulated glucose uptake. Biochemical Journal 352 267-276.

Foran PGP, Fletcher LM, Oatey PB, Mohammed N, Dolly JO \& Tavare JM 1999 Protein kinase B stimulates the translocation of GLUT4 but not GLUT1 or transferrin receptors in 3T3-L1 adipocytes by a pathway involving SNAP-23, synaptobrevin-2, and/or cellubrevin. Journal of Biological Chemistry 274 28087-28095.

Gordon GW, Berry G, Liang XH, Levine B \& Herman B 1998 Quantitative fluorescence resonance energy transfer measurements using fluorescence microscopy. Biophysical Journal 74 2702-2713.

Gray A, Van Der Kaay J \& Downes CP 1999 The pleckstrin homology domains of protein kinase B and GRP1 (general receptor for phosphoinositides-1) are sensitive and selective probes for the cellular detection of phosphatidylinositol 3,4-bisphosphate and/or phosphatidylinositol 3,4,5-trisphosphate in vivo. Biochemical Journal 344 929-936. 
Griffin BA, Adams SR \& Tsien RY 1998 Specific covalent labeling of recombinant protein molecules inside live cells. Science $\mathbf{2 8 1}$ 269-272.

Haugh JM, Codazzi F, Teruel M \& Meyer T 2000 Spatial sensing in fibroblasts mediated by $3^{\prime}$ phosphoinositides. Journal of Cell Biology 151 1269-1280.

Heim R \& Tsien RY 1996 Engineering green fluorescent protein for improved brightness, longer wavelengths and fluorescence resonance energy transfer. Current Biology 6 178-182.

Hirose K, Kadowaki S, Tanabe M, Takeshima H \& Iino M 1999 Spatiotemporal dynamics of inositol 1,4,5-trisphosphate that underlies complex $\mathrm{Ca}^{2+}$ mobilization patterns. Science $\mathbf{2 8 4}$ $1527-1530$.

Houtsmuller AB \& Vermeulen W 2001 Macromolecular dynamics in living cell nuclei revealed by fluorescence redistribution after photobleaching. Histochemistry and Cell Biology 115 13-21.

Kops GJ \& Burgering BMT 1999 Forkhead transcription factors: new insights into protein kinase B (c-akt) signaling. Journal of Molecular Medicine 77 656-665.

Mahajan NP, Linder K, Berry G, Gordon GW, Heim R \& Herman B 1998 Bcl-2 and Bax interactions in mitochondria probed with green fluorescent protein and fluorescence resonance energy transfer. Nature Biotechnology 16 547-552.

Mahajan NP, Harrison-Shostak DC, Michaux J \& Herman B 1999 Novel mutant green fluorescent protein protease substrates reveal the activation of specific caspases during apoptosis. Chemistry and Biology 6 401-409.

Matz MV, Fradkov AF, Labas YA, Savitsky AP, Zaraisky AG, Markelov ML \& Lukyanov SA 1999 Fluorescent proteins from nonbioluminescent Anthozoa species. Nature Biotechnology 17 969-973

Mitra RD, Silva CM \& Youvan DC 1996 Fluorescence resonance energy transfer between blue-emitting and red-shifted excitation derivatives of the green fluorescent protein. Gene 173 13-17.

Miyawaki A, Llopis J, Heim R, McCaffrey JM, Adams JA, Ikura M \& Tsien RY 1997 Fluorescent indicators for $\mathrm{Ca}^{2+}$ based on green fluorescent protein. Nature 388 882-887.

Monosov EZ, Wenzel TJ, Luers GH, Heyman JA \& Subramani S 1996 Labeling of peroxisomes with green fluorescent protein in living P-pastoris cells. Journal of Histochemistry and Cytochemistry 44 581-589.

Ng T, Squire A, Hansra G, Bornancin F, Prevostel C, Hanby A, Harris W, Barnes D, Schmidt S, Mellor H, Bastiaens PI \& Parker PJ 1999 Imaging protein kinase C alpha activation in cells. Science 283 2085-2089.

Oatey PB, Venkateswarlu K, Williams AG, Fletcher LM, Foulstone EJ, Cullen PJ \& Tavaré JM 1999 Confocal imaging of the subcellular distribution of phosphatidylinositol 3,4,5-trisphosphate in insulin- and PDGF-stimulated 3T3-L1 adipocytes. Biochemical Journal 344 511-518.

Perez F, Diamantopoulos GS, Stalder R \& Kreis TE 1999 CLIP-170 highlights growing microtubule ends in vivo. Cell 96 517-527.

Peyrollier K, Hajduch E, Gray A, Litherland GJ, Prescott AR, Leslie NR \& Hundal HS 2000 A role for the actin cytoskeleton in the hormonal and growth-factor-mediated activation of protein kinase B. Biochemical Journal 352 617-622.

Pollok BA \& Heim R 1999 Using GFP in FRET-based applications. Trends in Cell Biology 9 57-60.

Presley JF, Cole NB, Schroer TA, Hirschberg K, Zaal KJ \& Lippincott-Schwartz J 1997 ER-to-Golgi transport visualized in living cells. Nature 389 81-85.

Rain J-C, Selig L, De Reuse H, Battaglia V, Reverdy C, Simon S, Lenzen G, Petel F, Wojcik J, Schä chter V, Chemama Y, Labigne A \& Legrain P 2001 The protein-protein interaction map of Helicobacter pylori. Nature 409 211-215.

Rucktaschel AK, Granner DK \& Christ B 2000 Regulation by glucagon (cAMP) and insulin of the promoter of the human phosphoenolpyruvate carboxykinase gene (cytosolic) in cultured rat hepatocytes and in human hepatoblastoma cells. Biochemical Journal 352 211-217.

Sorkin A, McClure M, Huang F \& Carter R 2000 Interaction of EGF receptor and grb2 in living cells visualized by fluorescence resonance energy transfer (FRET) microscopy. Current Biology 10 1395-1398.

Stauffer TP, Ahn S \& Meyer T 1998 Receptor-induced transient reduction in plasma membrane $\operatorname{PtdIns}(4,5) \mathrm{P}_{2}$ concentration monitored in living cells. Current Biology 8 343-346.

Storrie B, White J, Rottger S, Stelzer EH, Suganuma T \& Nilsson T 1998 Recycling of Golgi-resident glycosyltransferases through the ER reveals a novel pathway and provides an explanation for nocodazole-induced Golgi scattering. Journal of Cell Biology 143 $1505-1521$

Takaishi H, Konishi H, Matsuzaki H, Ono Y, Shirai Y, Saito N, Kitamura T, Ogawa W, Kasuga M, Kikkawa U \& Nishizuka Y 1999 Regulation of nuclear translocation of forkhead transcription factor AFX by protein kinase B. PNAS 96 11836-11841.

Tenjinbaru K, Furuno T, Hirashima N \& Nakanishi M 1999 Nuclear translocation of green fluorescent protein-nuclear factor kappaB with a distinct lag time in living cells. FEBS Letters 444 1-4.

Tsien RY 1998 The green fluorescent protein. Annual Review of Biochemistry 67 509-544.

Tsien RY 1999 Rosy dawn for fluorescent proteins. Nature Biotechnology 17 956-957.

Tyas L, Brophy VA, Pope A, Rivett AJ \& Tavaré JM 2000 Rapid caspase-3 activation during apoptosis revealed using fluorescence-resonance energy transfer. EMBO Reports 1 266-270.

Vanhaesebroeck B \& Alessi DR 2000 The PI3K-PDK1 connection: more than just a road to PKB. Biochemical Journal 346 561-576.

Varnai P, Rother KI \& Balla T 1999 Phosphatidylinositol 3-kinase-dependent membrane association of the Bruton's tyrosine kinase pleckstrin homology domain visualized in single living cells. Journal of Biological Chemistry 274 10983-10989.

Venkateswarlu K, Gunn-Moore F, Oatey PB, Tavaré JM \& Cullen PJ 1998 a Nerve growth factor- and epidermal growth factor-stimulated translocation of the ADP-ribosylation factor-exchange factor GRP1 to the plasma membrane of PC12 cells requires activation of phosphatidylinositol 3-kinase and the GRP1 pleckstrin homology domain. Biochemical Journal 335 139-146.

Venkateswarlu K, Oatey PB, Tavaré JM \& Cullen PJ 1998b Insulindependent translocation of ARNO to the plasma membrane of adipocytes requires phosphatidylinositol 3-kinase. Current Biology 8 463-466.

Venkateswarlu K, Gunn-Moore F, Tavaré JM \& Cullen PJ 1999 EGFand NGF-stimulated translocation of cytohesin-1 to the plasma membrane of PC12 cells requires PI 3-kinase activation and a functional cytohesin-1 PH domain. Journal of Cell Science 112 1957-1965.

Wacker I, Kaether C, Kromer A, Migala A, Almers W \& Gerdes HH 1997 Microtubule-dependent transport of secretory vesicles visualized in real time with a GFP-tagged secretory protein. Journal of Cell Science 110 1453-1463.

van der Wal J, Habets R, Varnai P, Balla T \& Jalink K 2001 Monitoring phospholipase $\mathrm{C}$ activation kinetics in live cells by FRET. Journal of Biological Chemistry 276 15337-15344.

Watton SJ \& Downward J 1999 Akt/PKB localisation and 3' phosphoinositide generation at sites of epithelial cell-matrix and cell-cell interaction. Current Biology 9 433-436.

Xu X, Gerard AL, Huang BC, Anderson DC, Payan DG \& Luo Y 1998 Detection of programmed cell death using fluorescence energy transfer. Nucleic Acids Research 26 2034-2035.

Zaccolo M, De Giorgi F, Cho CY, Feng L, Knapp T, Negulescu PA, Taylor SS, Tsien RY \& Pozzan T 2000 A genetically encoded, fluorescent indicator for cyclic AMP in living cells. Nature Cell Biology 2 25-29.

Received 16 February 2001

Accepted 24 April 2001 